\title{
EchoGéo
}

36 | 2016

Stratégies de villes et "modèles urbains"

\section{La stratégie de la « smart city » au Japon : expérimentations nationales et circulations globales}

Raphaël Languillon-Aussel, Nicolas Leprêtre and Benoit Granier

\section{(2) OpenEdition}

Journals

Electronic version

URL: https://journals.openedition.org/echogeo/14598

DOI: $10.4000 /$ echogeo.14598

ISSN: 1963-1197

Publisher

Pôle de recherche pour l'organisation et la diffusion de l'information géographique (CNRS UMR 8586)

Electronic reference

Raphaël Languillon-Aussel, Nicolas Leprêtre and Benoit Granier, "La stratégie de la « smart city » au Japon : expérimentations nationales et circulations globales", EchoGéo [Online], 36 | 2016, Online since 30 June 2016, connection on 10 August 2021. URL: http://journals.openedition.org/echogeo/14598 DOI: https://doi.org/10.4000/echogeo.14598

This text was automatically generated on 10 August 2021

EchoGéo est mis à disposition selon les termes de la licence Creative Commons Attribution - Pas d'Utilisation Commerciale - Pas de Modification 4.0 International (CC BY-NC-ND) 


\title{
La stratégie de la « smart city » au Japon : expérimentations nationales et circulations globales
}

\author{
Raphaël Languillon-Aussel, Nicolas Leprêtre and Benoit Granier
}

\section{L'irruption du « smart » en Asie orientale et la spécificité de l'approche japonaise}

1 On observe depuis plusieurs années une multiplication des discours et des expérimentations sur les «smart cities » dans le monde entier. Près du tiers des projets en cours sont localisés en Asie, à l'exemple de celui de Songdo en Corée du Sud (Coll, 2014). Dans le sillage des stratégies d'entreprises comme IBM, Hitachi, Cisco ou Toshiba, les plus présentes sur le marché, les villes mondiales affichent désormais leur prétention à devenir (plus) «smart ». L'ensemble des services urbains serait concerné par ce processus, qu'il s'agisse de l'optimisation de la gestion des ressources, des intrants ou des sortants («smart grid»), des déplacements («smart mobility»), des relations sociales ("smart community») voire des citadins eux-mêmes («smart citizens»). La "smart city» serait-elle alors une nouvelle injonction pour les grandes métropoles inscrites dans la compétition et la circulation globales des modèles?

2 Les définitions des "smart cities» sont encore imprécises. Michael Batty les conçoit comme des villes structurées par la gestion instantanée des big data, ou méta-données, issues de la technologisation des espaces urbains et des réseaux ${ }^{1}$. Pour lui, les villes deviennent «smart» lorsqu'elles permettent le recours au traitement informatique simultané de l'information afin de gérer et d'anticiper la dynamique des espaces, des réseaux et des populations. La "smart city» repose ainsi sur l'usage massif de l'informatique, de capteurs, de super-calculateurs et de l'Internet, qui permettent de connaître et de gérer la ville sur le très court terme (Batty, 2012).

3 Les recherches sur les "smart cities " privilégient très majoritairement des entrées techniques et managériales (Meijer et Rodriquez Bolivar, 2014), alors que les approches 
par les sciences sociales sont encore émergentes. Une partie des recherches consiste à analyser l'origine de ces systèmes techniques (Picon, 2013) et l'impact de la multiplication exponentielle de données dans la production urbaine. L'entrée par les acteurs est encore relativement peu explorée en dehors du questionnement portant sur les modes de «bonne gouvernance» (Chourabi et al, 2012 ; Scholl et Scholl, 2014). De même, les postures critiques sur l'objet et le projet de la "smart city" sont encore relativement peu nombreuses dans le monde académique. Tandis que certains chercheurs de notoriété internationale ont exprimé leur méfiance (Sennett, 2012; Sassen, 2011), plusieurs études signalent leurs multiples dangers (Galdon-Clavell, 2013), en particulier pour la gouvernance démocratique (Gurstein, 2014), et avertissent de la mainmise du secteur privé (Söderström et al, 2014). Aucune n'interroge la notion de « modèle » global, asiatique, ou encore national, entendu comme « image de la ville » à la fois « exemplaire » et « reproductible » (Choay 1965, p. 16), ce que Max Weber appelle par ailleurs l'idéal-type.

$4 \mathrm{Au}$ Japon, les projets financés par le gouvernement sont dénommés «smart communities", même si certaines d'entre elles, tout comme d'autres projets privés, utilisent aussi l'appellation de "smart cities $»^{2}$. Un programme national a été lancé en 2010 par le Ministère de l'Economie, du Commerce et de l'Industrie (METI) et porte sur quatre sites pilotes (Granier, Lecler, Leprêtre, 2015). Des initiatives privées associant des collectivités locales l'ont suivi.

5 Alors que les municipalités sont perçues comme les acteurs montant de la gouvernance urbaine des grandes métropoles (Acuto, 2013), expliquant l'importance des réseaux de villes dans la circulation des modèles et des bonnes pratiques (Peyroux, 2014), l'État japonais apparaît comme l'acteur déterminant dans les "smart cities", ce qui inscrit les analyses dans la continuité des recherches sur l'action publique japonaise. Il s'agit ainsi de comprendre dans quelle mesure l'émergence des «smart cities " s'inscrirait non pas dans une logique de réseau de villes mais dans un modèle de développement japonais, dit développementalisme (Johnson, 1982), ce qui les rendrait uniques non pas dans leur forme mais dans leur genèse et leur gouvernance.

6 À ce titre, on pourrait se demander si, plus que le modèle, d'autres outils d'analyse théorique comme la grammaire de la sociologie pragmatique (Lemieux, 2000; Boltanski, 2006) ne seraient pas plus opérants pour décrire les "smart cities" japonaises. L'objectif de cet article est ainsi d'analyser l'approche japonaise, d'interroger ses spécificités, d'évaluer son exportabilité et de discuter son éventuelle constitution en « modèle » du «smart ».

7 Ce travail de recherche est issu de plusieurs phases de terrain dans plus d'une dizaine de «smart cities » au Japon, en France et en Chine au cours desquelles ont été menés des entretiens semi-directifs en français, en anglais et en japonais auprès d'acteurs publics et privés clés. La visite commentée des sites, associée à un travail bibliographique sur des sources primaires et des statistiques a permis de préciser l'approche qualitative (rapports, projets, comptesrendus internes des municipalités et de certaines entreprises, dont Toshiba). Il vient combler un manque de la recherche occidentale mais également japonaise sur l'analyse des "smart cities» en sciences humaines et sociales. La recherche japonaise sur le «smart» (grids, cities ou communities) a surtout été menée par des ingénieurs et des chercheurs provenant des sciences dures, si bien que les chercheurs japonais en sciences sociales qui s'y sont intéressés sont encore peu 
nombreux (Kudo et Granier, 2015 ; Kudo, 2013; Shiroyama et Kajiki, 2013 ; Hosono, 2000, 2013).

\section{«Smart », nouvelle grammaire de la production urbaine au Japon}

8 Le tournant des années 2010 a vu se propager le recours au «smart» dans les discours portant sur l'urbain, au Japon comme dans les autres pays industrialisés. Jusqu'alors, le vocable concernait essentiellement les innovations issues du web 3.0, ou Internet des objets (smartphone par exemple). La grammaire du «smart » a ainsi gagné l'urbain du fait essentiellement des grandes entreprises « IT », comme Google ou encore IBM et son programme de «Smarter City» lancé en 2008.

9 La sociologie pragmatique a défini la grammaire ${ }^{3}$ comme un ensemble de règles, de pratiques, de représentations, références et comportements communs qui font sens pour un certain nombre d'acteurs et permettent leur coordination à moindre frais. La grammaire permet à une communauté ou un groupe d'acteurs de juger, prendre des décisions et agir de concert sans que ne soient véritablement nécessaires des structures institutionnelles d'encadrement ou de coordination ${ }^{4}$ (Lemieux, 2000 ; Boltanski, 2006 ; Lemieux, 2009). Le "smart », plus qu'un modèle, se structure en grammaire nouvelle de la production et de la gestion des espaces urbains globalisés, dont les codes et les procédés doivent être analysés.

\section{Le «smart » et les nouveaux acteurs de l'urbain au Japon}

Le recours croissant à la grammaire de la « smart city » s'explique par une succession de mutations au Japon, que l'on retrouve dans un grand nombre de pays développés. On peut en lister quatre (illustration 1 ) :

1. L'évolution mature de la population est responsable, avec le vieillissement, à la fois d'un plus haut niveau de vie général mais aussi de l'apparition de nouveaux besoins en termes de prise en charge des personnes (Buhnik, 2010; Languillon, 2014). Haut niveau de vie et besoins spécifiques rendent possible la mise en place d'immeubles et de logements intelligents, qui constituent l'unité de base des «smart cities » japonaises, point qui ne se retrouve pas aussi fortement impliqué dans d'autres pays.

2. L'essoufflement de la politique de renaissance urbaine et l'impossible inflation des prix fonciers et immobiliers rendent nécessaire la relance de la création de valeurs au Japon, en particulier à Tokyo (Aveline, 2008; Languillon, 2013). La «smart city», et son très fort contenu d'innovations technologiques, permet non seulement de dynamiser le tissu économique mais aussi de faire monter les prix immobiliers.

3. D'une façon plus générale, pour l'ensemble des pays développés, l'arrivée sur le marché d'une série d'innovations à très fort contenu technologique que seules les économies les plus avancées du monde ont réussi, pour le moment, à mettre en place, rend nécessaire de trouver de nouveaux débouchés en raison de leur cycle-produit très court et de leur arrivée rapide à maturité. Fortement aidée au Japon par l'État, en particulier en ce qui concerne les réseaux informatiques et les réseaux de distribution énergétique («smart» grid), la «smart city » permet d'articuler entre eux ces cycles d'innovations appliqués à l'urbain.

4. Enfin, de nouveaux acteurs sont arrivés sur le marché de la fabrique et de la gestion urbaines suite à la diversification de leurs activités, et ce de façon concomitante au Japon, en Corée du Sud, à Taïwan, comme dans les pays occidentaux (tableau 1). Historiquement, on 
trouve les acteurs immobiliers dits classiques, que Isabelle Baraud-Serfaty (2011) appelle les «purs urbains ». Ils abordent l'urbain comme leur cœur de métier traditionnel. Dans cette catégorie se trouvent les promoteurs immobiliers ou les entreprises de services. À leurs côtés se tiennent les "urbains de fait ", ceux dont l'urbain relève des effets collatéraux de leurs activités, comme pour les commerciaux ou les « déclencheurs d'urbanité » (les grands magasins, les complexes de cinéma, les shopping mall). Au Japon, les "purs urbains » qui participent directement aux opérations d'urbanisation sont traditionnellement de trois types: les compagnies immobilières (fudôsan) des conglomérats (les célèbres keiretsu), les compagnies de chemin de fer privées (ôtemintetsu), et les entreprises de la construction (zenekon).

11 Depuis les années 1980, les acteurs issus de la finance ont fait irruption dans le champ de la fabrique urbaine, par le biais de la financiarisation de l'immobilier, constituant une première vague d'acteurs nouveaux ${ }^{5}$. Dans les années 1990 et 2000 , une deuxième vague de nouveaux acteurs, dits " opportunistes urbains », fait irruption dans le champ de l'urbain suite à l'évolution stratégique de leurs activités. L'entrée privilégiée correspond souvent à l'énergie, à la captation de valeur (Disney), ou à l'intégration vers l'amont (Eiffage, Vinci). Au Japon, ces acteurs nouveaux ont cela de particulier qu'ils correspondent en fait à des membres de coalitions historiques dont les entreprises les plus à l'écart de l'urbain finissent progressivement par pénétrer ce nouveau champ d'activité en raison de leurs relations et du partage d'intérêts stratégiques établis de longue date avec des acteurs aux activités plus directement urbaines ${ }^{6}$.

Illustration 1- Les quatre grandes mutations qui expliquent l'apparition des villes intelligentes au Japon.

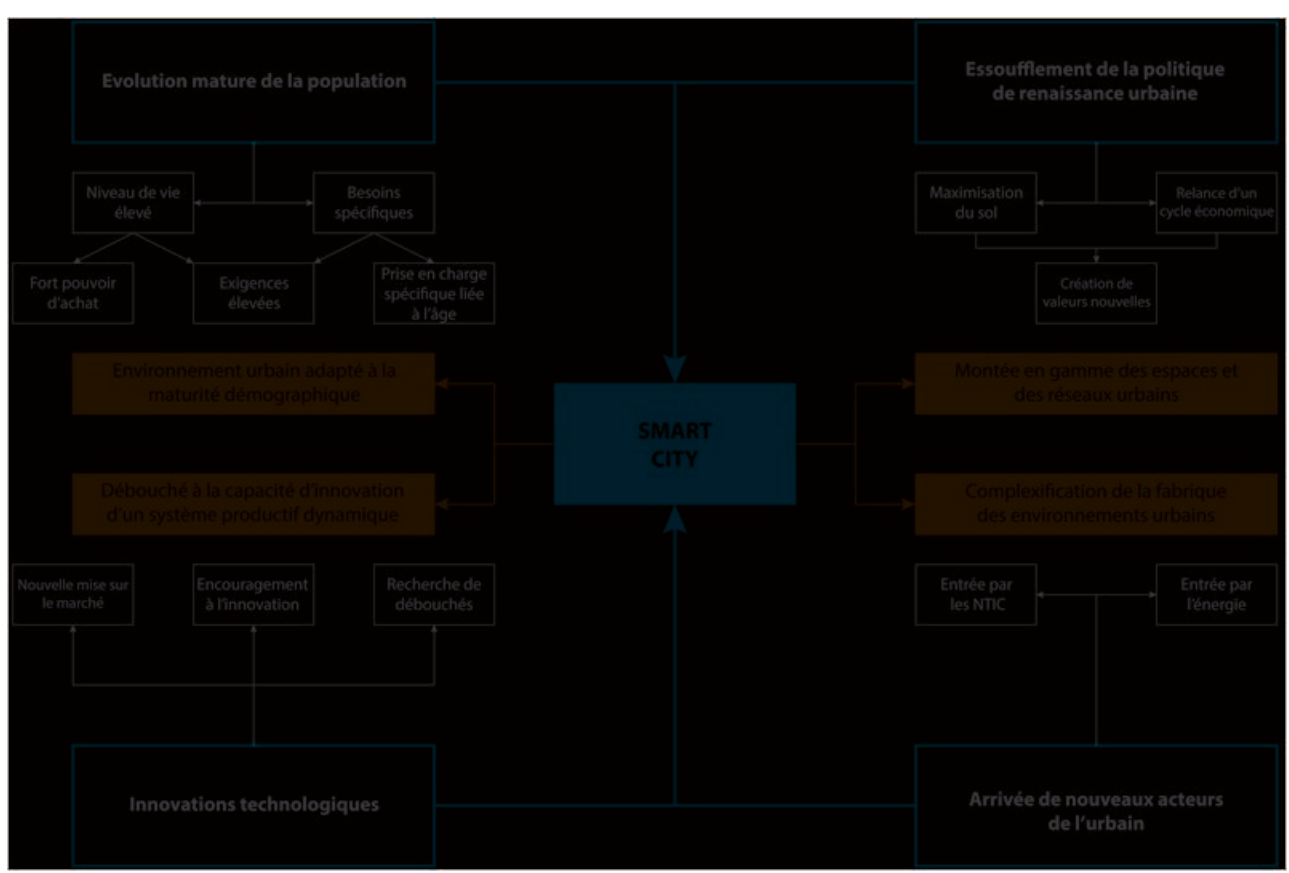

Source : Raphaël Languillon-Aussel, 2015.

12 Enfin, constituant une troisième et ultime vague, on trouve ce qu'Isabelle BaraudSerfaty appelle les " urbains de demain ». Issus des NTIC, ils pénètrent la ville par le biais de l'information, comme par exemple Google, IBM, DOCOMO, SoftBank, Toshiba. Au Japon ils se sont associés aux acteurs de la mobilité (Toyota Motor, Nissan Motor) 
qui s'investissent également dans une approche dite "smart» des services de transports ${ }^{7}$.

Tableau 1 - Synthèse des différents types d'acteurs qui interviennent dans la fabrique et la gestion des environnements urbains

\begin{tabular}{|c|c|c|c|c|}
\hline \multirow{2}{*}{ Catégorie } & \multirow{2}{*}{$\begin{array}{l}\text { Positionnement par } \\
\text { rapport à l'urbain }\end{array}$} & \multirow{2}{*}{ Sous-catégorie } & \multicolumn{2}{|c|}{ Exemples } \\
\hline & & & France et Europe & Japon \\
\hline Les purs urbains & Urbain $=$ coeur de métier & $\begin{array}{l}\text { - Entreprises de limmobilier } \\
\text {-Entreprises de services urbains } \\
\text { - Investisseurs traditionnels }\end{array}$ & $\begin{array}{l}\text { - Nexity, Unibail } \\
\text { - Véolia, Suez, Saur } \\
\text { - Macquarie }\end{array}$ & $\begin{array}{l}\text { - Mitsui Fudôsan } \\
\text { - Tôkyû, Tôbu } \\
\text { - Mitsubishi UFG Trust } \\
\text { and Banking }\end{array}$ \\
\hline Les urbains de fait & $\begin{array}{l}\text { Urbain = effet collatéral des } \\
\text { activités du coeur de métier }\end{array}$ & $\begin{array}{l}\text { - Les grands utilisateurs } \\
\text { - Commerciaux } \\
\text { - Utilisateurs de bureaux } \\
\text { - Les grands investisseurs (de fait) } \\
\text { - Les déclencheurs d'urbanité }\end{array}$ & $\begin{array}{l}\text { - Auchan, Mc Do } \\
\text { - Renault, BNP, Michelin } \\
\text { - Axa, Generali } \\
\text { - Printemps, Cinémas }\end{array}$ & $\begin{array}{l}\text { - Daiei } \\
\text {-Sumitomo Mitsui Banking } \\
\text { Corporation } \\
\text { - Mitsukoshi, táminaru depáto } \\
\text { (Keio dpt store, Tökyũ dpt store, }\end{array}$ \\
\hline Les opportunistes urbains & $\begin{array}{l}\text { Urbain = résultat d'une } \\
\text { évolution stratégique }\end{array}$ & $\begin{array}{l}\text { - Intégration par l'amont } \\
\text { - Diversification } \\
\text { - Captation de valeur } \\
\text { - Entrée par l'énergie }\end{array}$ & $\begin{array}{l}\text { Eiffage, Vinci } \\
\text { ADP, OL Land } \\
\text { - Ikéa, Disney } \\
\text { Siemens, General Electrí }\end{array}$ & $\begin{array}{l}\text { - Disney (Oriental Land } \\
\text { Company) } \\
\text {-TEPCO, Toshiba }\end{array}$ \\
\hline Les urbains de demain & $\begin{array}{l}\text { Urbain = entrée par } \\
\text { l'information } \\
\text { et la communication }\end{array}$ & Les entreprises IT & Google, IBM, Cisco & $\begin{array}{l}\text { Google, Sony, Toshiba, } \\
\text { Panasonic... }\end{array}$ \\
\hline
\end{tabular}

Source : Isabelle Baraud-Serfaty, in Damon J., Denis E. et Strauch L., 2013, p.51.

\section{Le rôle de l'État dans l'émergence du « smart » japonais}

L'État joue un rôle majeur dans l'émergence du «smart » japonais, en raison de son approche spécifiquement « développementaliste ». Proposée par Chalmers Johnson en 1982, la notion d'État développementaliste renvoie à de forts liens entre l'administration, les acteurs privés et les politiciens dans l'élaboration et la conduite des politiques publiques favorisant les innovations technologiques.

L'approche développementaliste de l'État japonais a fait l'objet de nombreux débats au sein de la littérature (Leprêtre, 2015), notamment sur ce qui constitue sa spécificité. Le concept a été développé par $\mathrm{Ch}$. Johnson pour décrire la stratégie de "rattrapage de l'Occident ", avec un accent sur la période d'après-guerre dans son étude et le rôle de l'administration dans ce processus. Outre des mécanismes d'intervention de l'État dans l'économie tout en favorisant le libre-marché et l'imprégnation des intérêts corporatistes au sein des appareils bureaucratiques (Stockwin, 1999), le développementalisme se distingue par un "principe d'activisme» partagé par l'ensemble des acteurs politico-économiques visant à promouvoir des secteurs d'activités prioritaires pour la compétitivité économique du pays (Thurbon, 2014, p. 73-74). Le secteur de l'informatique fait partie des priorités économiques depuis le plan MASUDA "pour une société informatique" dans les années 1970, suivi d'un ensemble de programmes nationaux de subventions aux technologies de pointe (Callon, 1995).

15 Ainsi, dans des années 1990 marquées par une fin de la stratégie de rattrapage, par la concurrence des pays asiatiques sur les nouveaux marchés japonais de technologies de pointe et par une crise économique qualifiée de "Décennie Perdue », cette approche développementaliste se manifeste par un intense soutien aux innovations 
technologiques environnementales, en particulier avec la promotion des écoinnovations ${ }^{8}$ dans les années 2000 . Ce soutien va prendre la forme, au tournant des années 2000 , d'une régionalisation des politiques d'innovation à travers des pôles de compétitivités ou «cluster » qui se combinent à d'autres politiques environnementales et urbaines. On assiste ainsi à une convergence des instruments de soutien à ces écoinnovations (Leprêtre, 2016).

Dans la transition des éco-innovations inspirées du développement durable au "smart», l'État a joué un rôle essentiel à deux niveaux: dans son soutien aux expérimentations locales et dans son encouragement à l'internationalisation, les deux étant intimement liés dans la logique développementaliste.

\section{Les initiatives publiques japonaises et le programme national}

17 À partir de 2009, le gouvernement japonais a progressivement mis en place un ensemble d'initiatives visant à promouvoir le développement et la mise en œuvre de technologies dites «smart». Les moyens par lesquels sont organisées l'identification des technologies à privilégier et leur introduction sont significatifs d'un modus operandi relativement classique pour le gouvernement japonais. Ce dernier a mis en place courant 2009 un ensemble de groupes de recherche regroupant des universitaires et des grandes firmes japonaises dans le but de déterminer les technologies clés à développer. C'est à partir de ces travaux que vingt-six technologies ont été sélectionnées en janvier 2010 pour un développement prioritaire afin de promouvoir leurs normes sur la scène internationale avec le soutien du gouvernement. Pour perpétuer le dialogue entre entreprises initié dans ces premiers groupes de réflexions, le METI a soutenu la création d'un Smart Community Forum en 2009, remplacé en 2010 par la Japan Smart Community Alliance (JSCA). Fondée à l'initiative du NEDO (New Energy and Industrial Technology Development Organization) et du METI, l'objectif de cette association est de créer un espace de dialogue entre firmes sur les normes internationales.

18 Au-delà de ces cercles de réflexion et d'échanges, le METI a mis en place quatre programmes majeurs visant à l'introduction de technologies «smart» au niveau local. Le plus significatif par son budget ( 40 milliards de yens au total) est appelé «Next Generation Energy and Social System ", ou encore "Smart community » et a été mis en place de 2010 à 2015 (illustration 2) ${ }^{9}$. Suite à un appel d'offre en janvier 2010, vingt collectivités ont candidaté avec des projets impliquant chacun un consortium d'entreprises. À l'issue d'une sélection à laquelle a participé un comité d'experts, quatre «smart communities » ont été sélectionnées en raison d'atouts bien distincts : la ville de Yokohama ${ }^{10}$ pour une expérimentation en milieu urbain dans une très grande ville (illustration 3), la ville de Toyota $^{11}$ comme ville de province avec un accent sur le transport, le département de Kyoto pour son projet au sein de la cité scientifique de Keihanna ${ }^{12}$ et une expérimentation dans une ville industrielle à Kitakyushu ${ }^{13}$. Après leur sélection, ces quatre collectivités et les entreprises impliquées ont rédigé avec le comité d'expert et le METI un master plan entre avril et août 2010, avant de débuter l'expérimentation.

19 À l'exception de Yokohama, dont l'expérimentation « smart » est plus large que le cadre $\mathrm{du}$ seul quartier, les programmes pilotes des quatre «smart communities " japonaises sont spatialement ciblés et réduits. La variété des quatre cas permet de tester divers 
éléments et innovations, sans prendre en considération toute la complexité des objets " villes ». Les expérimentations concernent ainsi 225 foyers à Toyota, 230 à Kitakyushu, 700 à Kyoto Keihanna et 4000 à Yokohama, qui est de loin l'initiative la plus vaste et la plus transversale du Japon. Chaque initiative est gouvernée par une collectivité locale (la municipalité, ou le département de Kyoto dans le cas de Keihanna) en association avec un consortium d'entreprises privées, et une ONG écologiste - Satoyama-okangaeru-kai - dans le cas de Kitakyushu.

D'abord centré sur le développement de technologies compétitives en vue d'un déploiement à l'international et la réduction des émissions de gaz à effet de serre, le programme de "smart communities» a subi une réorientation importante de ses objectifs suite à l'accident de la centrale de Fukushima de mars 2011 et l'arrêt de la production nucléaire au sein de l'archipel. La fragilité du réseau électrique, pourtant réputé solide, qui résulte de ces événements a incité les acteurs publics et privés à développer en priorité des systèmes de gestion de l'énergie et d'incitation à réduire la consommation en période de pic. L'accident de Fukushima a également mis à l'agenda la question des politiques énergétiques. Si l'énergie nucléaire reste un objet de controverse, surtout depuis le redémarrage de réacteurs en 2015 sous le gouvernement de Shinzo Abe, le soutien au développement de «smart cities » est présenté comme une orientation forte des stratégies économiques à venir : déjà présent dans la «Stratégie de Nouvelle Croissance » du Parti Démocrate Japonais au pouvoir en 2010, le soutien aux «smart grids» est réitéré dans la «Stratégie de Revitalisation du Japon » du Parti Libéral-Démocrate au pouvoir en 2013.

21 L'appel d'offre du METI a été l'occasion de signifier les attentes du ministère en termes de développement technologique, à la suite des vingt-six critères clés qu'il avait établis l'année précédente. Parmi les éléments constitutifs du programme, on trouve un déploiement «massif » d'énergies renouvelables, l'établissement de systèmes de gestion de l'énergie (Energy Management System ou EMS), l'introduction de systèmes de transport "nouvelle génération ", la mise en place d'innovations dans les modes de vie et l'implication de l'ensemble des parties prenantes (industries, commerces locaux, habitants).

22 Les systèmes de gestion d'énergie (EMS) s'articulent à plusieurs échelles : celle de la communauté avec le CEMS (Community Energy Management System), à l'échelle de l'immeuble (BEMS), de la maison (HEMS) et de l'usine (FEMS), généralement équipés d'un dispositif de visualisation de la consommation (tablette, afficheur mural). Chacun de ces systèmes de gestion de l'énergie est connecté au CEMS qui centralise l'ensemble des données et prévoit les pics de consommation en fonction des profils de consommation et des prévisions météorologiques. Le CEMS envoie alors une requête de " demande-réponse ", ou DR, incitant les foyers et les employés d'usine ou de bureaux à réduire leur consommation en période de pic en été et en hiver ${ }^{14}$. Diverses innovations des systèmes de transport sont également mises au point.

Les quatre «smart communities» labellisées par le gouvernement sont le terrain d'un ensemble varié d'expérimentations sociotechniques couvrant plusieurs secteurs d'activité (production énergétique, gestion des données, transport). Le contact avec la population, à travers sa réactivité face aux requêtes de DR ou encore dans ses choix de consommation énergétique ou de mobilité, est également une composante essentielle de la vérification dans des conditions « réelles » de ces technologies (Granier et Kudo, 2015). Cette diversité des technologies et systèmes sociaux interroge l'idée d'un modèle 
unique au Japon, même si les modalités "politiques » de mise en place sont les mêmes pour toutes les expérimentations.

\section{Illustration 2 - Les quatre projets de "smart community »}

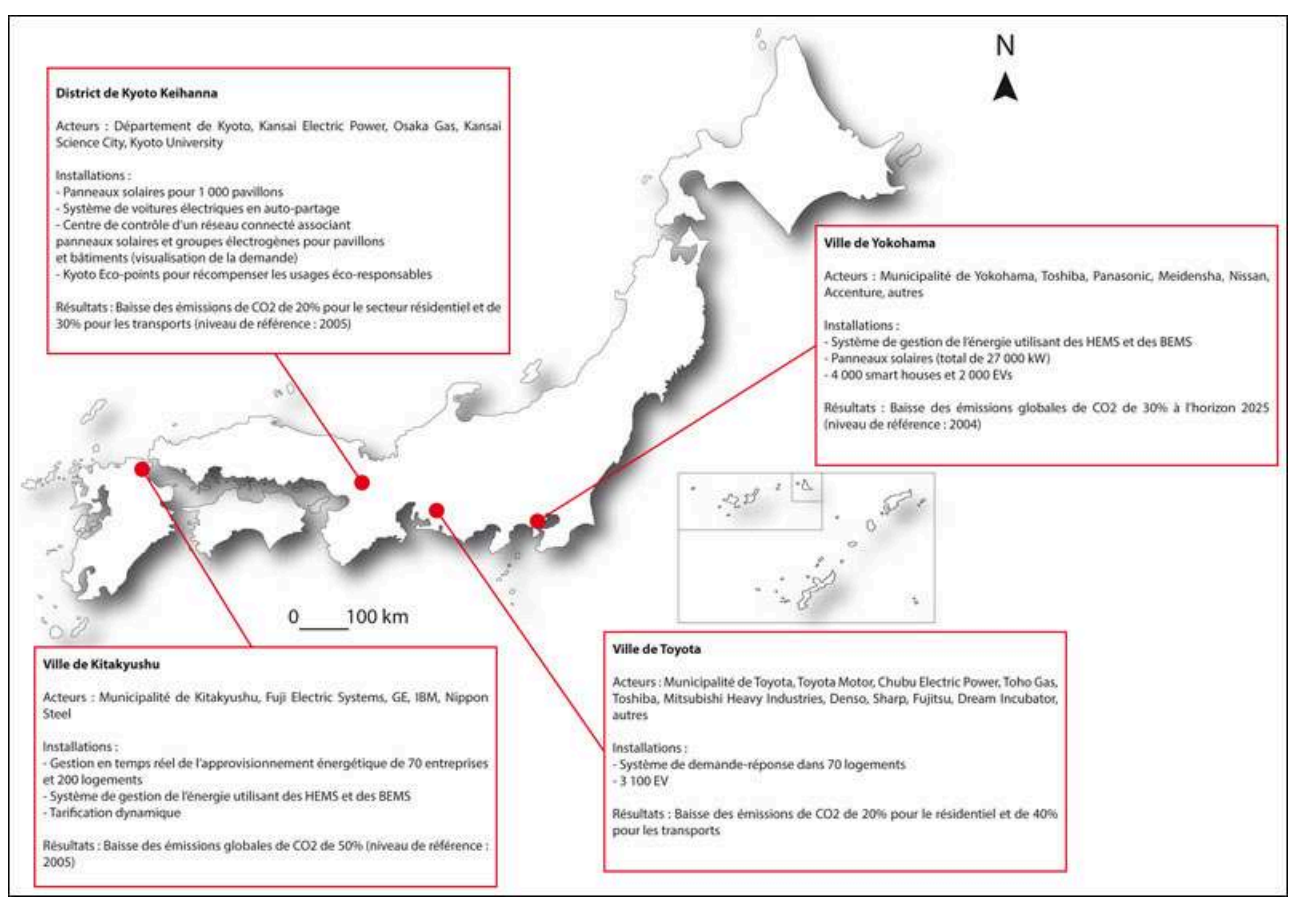

Source : Global Smart Grid Federation, 2014. Approaches to Smart Grid and Smart Community Deployment, 28 mai 2014, http://www.globalsmartgridfederation.org/2014/05/28/japans-approaches-to-smartgrid-and-smart-community-deployment/

Illustration 3 - Schéma de la « smart community » de Yokohama en 2012

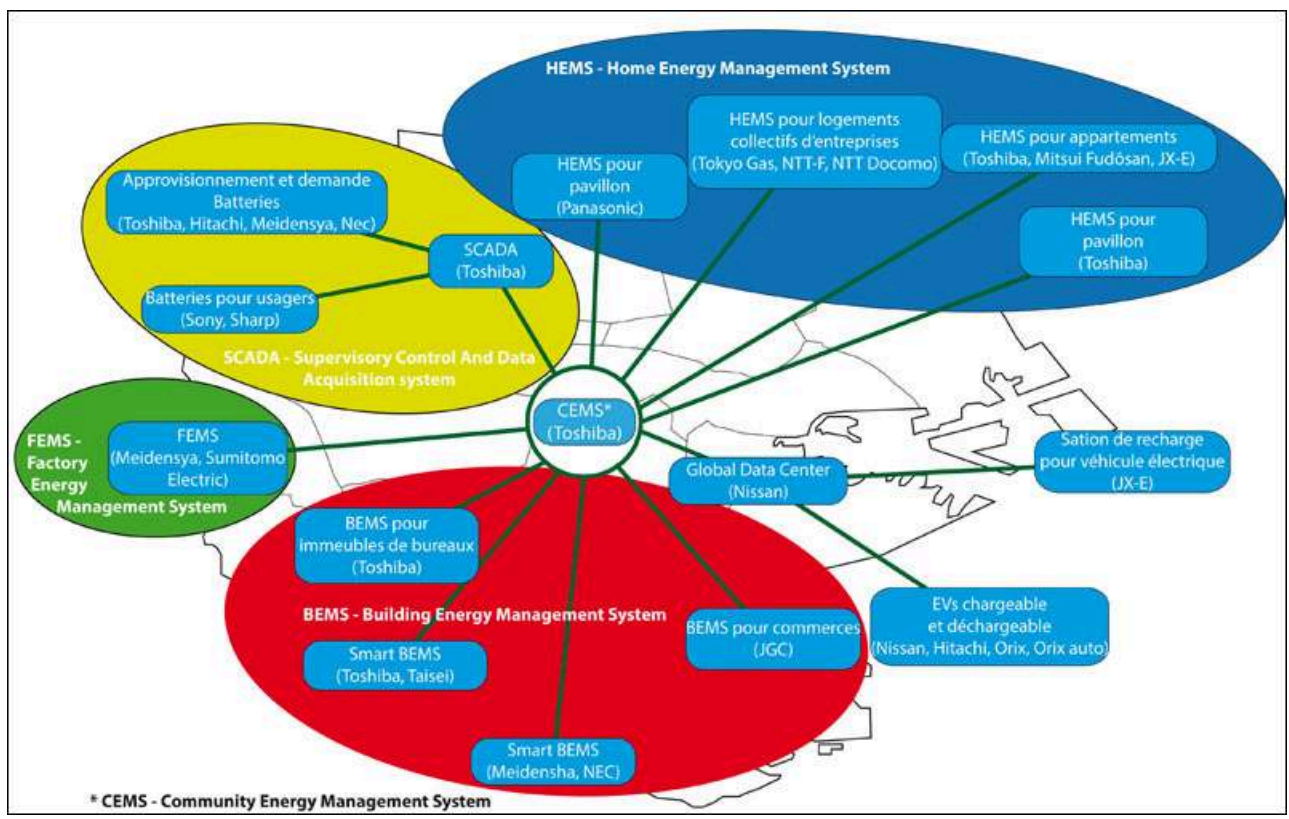

Source : Ville de Yokohama, 2012. "Next-Generation Energy and Social Systems Demonstration Areas", présentation du Climate Change Policy Headquarters lors de la Smart City Week, p. 4. 


\section{L'internationalisation du « smart » japonais}

le fait non pas des réseaux de villes mais de grands groupes privés, majoritairement des conglomérats, en partenariat avec le gouvernement ou dans des initiatives purement privées. Deux entreprises sont particulièrement actives: Toshiba et Hitachi. Elles interviennent sur l'ensemble des éléments constituant les environnements urbains : les réseaux, les équipements lourds, le matériel de travaux publics, les objets domestiques comme l'électronique ou l'électroménager. Hitachi a même été qualifié de "groupe industriel urbain total » en raison de ses ramifications dans toutes les dimensions de la production, de la gestion et de la consommation urbaines au Japon (Lorrain, 2004). Pour ces groupes, l'internationalisation des expérimentations offre de nombreux avantages, que l'on se propose d'analyser avec le cas de Toshiba.

\section{L'intérêt d'un grand groupe japonais pour le « smart »- le cas de Toshiba}

Toshiba est l'un des géants de l'électronique, de l'informatique et des télécommunications à l'échelle globale, au même titre qu'IBM. Au cours de l'année fiscale 2012 (d'avril 2012 à mars 2013), ses ventes ont totalisé 5800 milliards de yens, soit environ 64 milliards de dollars. $42 \%$ concernent les infrastructures dites « sociales » par le groupe (air conditionné, escalators, ascenseurs, turbines, panneaux solaires, usines thermiques au gaz), 23 \% les produits digitaux (écrans de télévision LED et LCD, notebooks, PC, tablettes, ordinateurs portables), $21 \%$ les produits électroniques (flash memory cards, circuits électriques), $8 \%$ les produits domestiques (ampoules LED, réfrigérateurs et machines à laver, piles alcalines), et $5 \%$ pour le reste des activités. Tous ces secteurs concernent des innovations mobilisables dans les «smart » devices, les «smart » buildings et les villes « intelligentes ».

Dans les années 2010 la stratégie de Toshiba consiste ainsi à produire des modèles viables de «smart cities " à partir d'une grande synthèse de l'ensemble de ses activités. Cette stratégie positionne le groupe comme l'un des leaders mondiaux des expérimentations « smart » à travers le monde.

La justification sociale mise en avant par Toshiba découle de l'identification de quatre grands enjeux auxquels la "smart city" tente de répondre de façon lucrative : l'enjeu environnemental, avec la hausse des émissions de polluants et de gaz à effet de serre, et le réchauffement global qui en résulte; les pressions sur la demande en énergie, en relation avec le développement; l'explosion du volume de données numériques et l'enjeu que leur traitement requiert; les mutations sociales, en particulier le vieillissement de la population japonaise, avec un souci de maintenir la qualité de vie. S'ajoutent également d'autres paramètres : l'arrivée à maturité d'un certain nombre d'innovations qui rend nécessaire un rebond et de nouveaux débouchés dans le secteur de l'électronique, de l'informatique, des télécommunications ; une pression politique en faveur de l'innovation et à la compétitivité des entreprises japonaises ; un niveau de vie élevé qui permet aux habitants d'absorber les innovations coûteuses du « smart ».

L'innovation majeure des "smart cities" développées par Toshiba repose sur une application du cloud computing à la gestion des espaces urbains. Appelé aussi informatique en nuage, le cloud computing utilise un réseau de serveurs informatiques

EchoGéo, 36 | 2016 
qui "dématérialise" l'infrastructure informatique pour offrir aux usagers plus de puissance de calcul et plus de possibilité de stockage de l'information. Les expérimentations à Yokohama sont, à cet égard, assez révélatrices de la stratégie adoptée par Toshiba: en investissant la gestion des données des BEMS par un «Integrated BEMS » par cloud et des batteries par le système SCADA ${ }^{15}$, Toshiba entend s'implanter dans une gestion des données à grande échelle intégrant une multitude de dispositifs techniques. Le développement de ces technologies s'inscrit donc dans une approche assez pragmatique selon laquelle Toshiba n'aura pas le contrôle de l'ensemble des dispositifs techniques, mais entend faciliter leur communication et la récolte des données par cloud.

L'intérêt de Toshiba pour les «smart cities» repose sur le fait qu'elles permettent d'articuler des milliards de données générées par les smart devices, les smart grid et les capteurs disséminés dans l'espace urbain, et donc permet un meilleur usage des métadonnées pour gérer la ville, ses besoins et ses contraintes en temps réel. Au final, la «smart city» de Toshiba articule deux grands domaines: les innovations énergétiques (production, acheminement, distribution, consommation); les innovations informationnelles fondées sur le cloud et gérées par ce dernier (data center, serveurs, réseaux, Internet, capteurs, GPS).

\section{L'enjeu de l'international pour les grands groupes japonais}

30 L'internationalisation des expérimentations japonaises du «smart» est le fait des grands groupes. À ce titre, Toshiba mène 35 projets dans le monde. Cette internationalisation peut être de deux ordres: l'exportation d'un modèle et d'un savoir-faire technologique, l'expérimentation à l'étranger pour réimporter au Japon un retour sur expérience. Pour l'heure, il s'agit surtout de la seconde option.

31 En effet, multiplier les initiatives à l'étranger est un enjeu fort pour les entreprises japonaises, et ce pour plusieurs raisons principales. L'accès futur aux marchés étrangers est un point important, qui se prépare dès à présent avec la mise en place de coopérations avec des entreprises nationales comme EDF, Véolia ou Engie en France. La recherche de co-financements de projets-pilotes avec des entreprises partenaires et des gouvernements étrangers permet également d'alléger les coûts de la recherche et de l'innovation technologique. La fertilisation croisée de technologies de pointe japonaises et étrangères est aussi un élément important. L'étude de la réaction des marchés tests, en particulier dans la réception et l'usage de technologies nouvelles dans des contextes socio-culturels variés, permet d'anticiper des rejets futurs. Les acteurs publics nationaux participent à cette stratégie d'internationalisation des firmes japonaises dans le domaine des «smart cities». Le NEDO a ainsi soutenu le développement de six démonstrateurs principaux, parallèles à l'établissement de nombreuses initiatives privées : Hawaii, Java en Indonésie, Los Alamos (Nouveau Mexique, USA), Manchester, Malaga, Lyon. Ces projets s'appuient sur les investissements lourds de compagnies japonaises : Toshiba, Mitsubishi Electric, Mitsubishi Motors.

32 Enfin, investir dans des projets tests à l'étranger permet également de mettre au point l'interopérabilité entre technologies et d'imposer ses propres normes à ses concurrents. Les protocoles de communication entre les différents éléments de la "smart city", qui ne sont pas encore arrêtés représentent un objet de compétitions entre les firmes japonaises et un point clé des expérimentations en cours. Au sein des 
maisons dites «smart ", la norme "Echonet Lite » de communication entre les appareils électroniques domestiques, promue par Toshiba, a finalement été adoptée avec le soutien du METI et a dû être reprise par les entreprises japonaises qui ne l'avaient pas jusqu'alors adoptées, comme Panasonic ${ }^{16}$. Du point de vue de la communication entre bâtiments, le protocole adopté par Toshiba ainsi que par plusieurs firmes américaines est appelé «Open $A D R$ », et l'entreprise s'en fait la promotrice au sein de l'organisation internationale en charge de la standardisation, la Commission Electronique Internationale (CEI ou IEC), et à travers le réseau international Open ADR Alliance.

Pour l'heure, Toshiba n'a pas encore développé de ville entièrement «smart ". L'entreprise teste dans des espaces pilotes des aspects partiels qui, mis bout-à-bout, explorent les différents aspects d'une hypothétique «smart city». Deux projets sont particulièrement aboutis et importants : celui de Yokohama au Japon et celui de Lyon Confluence ${ }^{17}$ en France, dont la forme technologique est quasi-identique - un CEMS reliés à des bâtiments et des systèmes de transport nouvelle génération. Le développement de démonstrateurs comme Yokohama doit donc également être compris dans cette logique de vérification des technologies en vue de leur standardisation internationale.

\section{Conclusion : Existe-t-il un modèle japonais de la « smart city»?}

34 Le programme national des Smart Communities lancé en 2010 par l'État rassemble la quasi-totalité des grandes entreprises japonaises ayant fait du «smart» un de leurs secteurs principaux d'activité pour les années à venir. Ainsi, malgré la persistance d'un certain nombre d'initiatives indépendantes et originales, il ne semble pas infondé de supposer que le modèle de "smart city" en voie de formation ressemble fortement à celui imaginé et désormais expérimenté dans les quatre «smart communities».

Pour le moment néanmoins, il semble prématuré de parler d'un modèle japonais de la "smart city", tant les initiatives sont encore balbutiantes et restreintes à quelques cas pilotes. Il serait plus juste de parler d'une grammaire naissante du «smart » appliquée à l'urbain. Si modèle il y a, ce serait plutôt dans la mise en place typiquement développementaliste des initiatives «smart » dans les espaces urbains japonais et leurs homologues expérimentaux étrangers dans lesquels les entreprises japonaises jouent un rôle prépondérant. Ainsi, plus que les réseaux de villes, deux types d'acteurs priment : l'État et son approche développementaliste, et les grandes multinationales spécialisées dans les NTIC.

Si l'avance du Japon dans les expérimentations des "smart cities» est avérée, et explique également les partenariats noués à l'étranger, comme à Lyon Confluence par exemple, il n'est pas dit que l'avance technologique du Japon dans le domaine des innovations mobilisées soit ni si importante, ni si spécifique. Il est ainsi saisissant de voir les similitudes entre les initiatives japonaises et les initiatives coréennes et chinoises. Le transport «intelligent» de Toyota rappelle celui testé à Shanghai par l'Académie chinoise des sciences, sur le campus du Zhangjiang High Tech Park. La ville nouvelle privée "intelligente» de Kashiwa no Ha rappelle celle de Songdo, la dimension sécuritaire en moins. 
L'autre inconnue des expérimentations "smart » au Japon concerne le positionnement habitant et le type de société et de sociabilité que l'innovation est en train de créer. D'une part, les expérimentations mises en place au Japon se fondent pour l'instant exclusivement sur le volontariat. À partir du moment où les habitants qui testent les technologies et le mode de vie «smart » sont volontaires, l'étude de leur comportement ne permet pas de se faire une idée du positionnement de l'ensemble de la population : résistance, crainte, rejet, usages... sont autant de points essentiels qui ne pourront être abordés que lors des phases futures de généralisation du «smart ». D'autre part, les études comportementales menées pour le compte du METI sont principalement axées sur la consommation d'énergie des ménages, tandis qu'il s'agit logiquement pour les entreprises de stratégie marketing. Les questions du changement des modes de vie au sens fort ou de l'appropriation des nouvelles technologies par les habitants sont ainsi encore très peu analysées. Pour le moment, il ne s'agit pas de villes au sens où les programmes ne concernent que des espaces réduits pilotes, programmes à l'approche par ailleurs très partielle centrée sur un élément parmi la multitude qui fonde la complexité des systèmes urbains.

\section{BIBLIOGRAPHY}

Acuto M., 2013. Global Cities, Governance and Diplomacty. The Urban Link, Routledge New Diplomacy Studies, $220 \mathrm{p}$.

Aveline N., 2008. Immobilier - l'Asie, la bulle et la mondialisation. Paris, CNRS Editions, 320 p.

Baraud-Serfaty I., 2011. La nouvelle privatisation des villes. Esprit, 2011-3-4, pp. 149-167.

Batty M., 2013. Big Data, Smart Cities and City Planning. Dialogues in Human Geography, n 3-3, p. 274-279

Boltanski L., 2006. Préface. In Nachi M., Introduction à la sociologie pragmatique. Paris, Armand Colin, coll. Cursus, p. 9-16.

Buhnik S., 2010. From shrinking cities to toshi no shukushô: identifying patterns of urban shrinkage in the Osaka Metropolitan Area. Berkeley Planning Journal, n²3-1, p. 132- 155.

Callon S., 1995. Divided Sun : MITI and the Breakdown of Japanese High-Tech Industrial Policy, 1975-1993. Stanford, California university press.

Chourabi H., Nam T., Walker S. J., Gil-Garcia R., Mellouli S., Nahon K., Pardo T.A., et Scholl H.J., 2012. Understanding Smart Cities: An Integrative Framework. Communication at the 45th Hawaii International Conference on System Science.

Choay F., 1965. L’Urbanisme, utopie et réalité. Paris, Seuil.

Coll J.M., 2014. Beyond Smart Cities: It's Time for Urban Sustainable Development. Notes Internacionals $C I D O B, \mathrm{n}^{\circ}$ 92, juin 2014.

Damon J., Denis E., Strauch L., 2013. Smart cities : efficaces, innovantes, participatives, comment rendre la ville plus intelligente ? Institut de l'Entreprise, $177 \mathrm{p}$.

EchoGéo, 36 | 2016 
DeWit A., 2014. Japan's Radical Energy Technocrats: Structural Reform Through Smart Communities, the Feed-in Tariff and Japanese-Style "Stadtwerke". The Asia-Pacific Journal 12, $n^{\circ} 48(2)$.

DeWit A., Tetsunari I., Kaneko M., 2012. Fukushima and the political economy of power policy in Japan. In J. Kingston (eds.), Natural Disaster and Nuclear Crisis in Japan. Response and recovery after Japan's 3/11. New-York, Routledge, p. 156-171

Galdon-Clavell G., 2013. (Not so) smart cities?: The drivers, impact and risks of surveillanceenabled smart environments. Science and Public Policy, Vol. 40, nº 6, p. 717-723.

Granier B., 2016. Changer les modes de vie pour réduire les émissions : émergence et limites des politiques japonaises de changement des comportements. Japan Analysis, ${ }^{\circ} 39$.

Granier B., 2015. L'expérimentation sociotechnique fondée sur les sciences comportementales : Un instrument au service de la production de l'acceptabilité sociale ?. Vertigo - la revue électronique en sciences de l'environnement [En ligne], vol. 15, n³, DOI : 10.4000/vertigo.16695 ; URL : http://vertigo.revues.org/16695

Granier B., Kudo H., 2015. How are Citizens Involved in Smart Cities? Analysing Citizen Participation in Japanese Smart Communities. Information Polity, DOI: 10.3233/IP-150367; URL: http://content.iospress.com/articles/information-polity/ip-1-ip367

Granier B., Lecler Y., Leprêtre N., 2015. Expérimenter les systèmes énergétiques de demain : les « smart communities » japonaises. Communication lors du colloque «Sciences Sociale et Transition Énergétiques », Grenoble, 28-29 mai 2015.

Gurstein M., 2014. Smart Cities vs. Smart Communities: Empowering Citizens not Market Economics. The Journal of Community Informatics, Vol. 10, $\mathrm{n}^{\circ}$ 3. URL : http://ci-journal.net/ index.php/ciej/article/view/1172/1117 - consulté le 7 janvier 2015.

Hosono S., 2013. Machizukuri no sumâto kakumei (La révolution intelligente de l'urbanisme participatif). Tokyo, Jijitsûshinsha, 295 p.

Hosono S., 2000. Sumâto komyuniti. Toshi no saisei kara nihon no saisei he (Smart community. De la renaissance des villes à la renaissance du Japon). Tokyo, Chûô University Press, 275 p.

Johnson Ch., 1982. MITI and the Japanese Miracle : The Growth of Industrial Policy, 1925-1975. Stanford, Stanford University Press, $412 \mathrm{p}$.

JSCP (Japan Smart City Portal), 2012. What are Smart Cities? Japan Smart City Portal Website. http://jscp.nepc.or.jp/article/jscp/20120817/319942/

Kudo Hiroko, 2013. « Quality of Life » and « resilience »: Japanese smart city projects after the 3.11 Great East Japan Earthquake. Paper for the 2013 EGPA Annual Conference, 11-13 September, Edinburgh, Scotland.

Languillon-Aussel, R. 2015. Kashiwa no Ha: laboratoire urbain pour les nouveaux enjeux des populations matures. Urbia, ${ }^{\circ} 18$, p. 125-146

Languillon-Aussel, R., 2014. Tokyo, ville globale mature. Essai de proposition critique sur la maturité urbaine au Japon. Urbia, numéro spécial $n^{\circ} 2$, Lausanne.

Languillon-Aussel R., 2013. Crise immobilière et privatisation de l'aménagement à Tokyo. Métropolitiques, URL : http://www.metropolitiques.eu/Crise-immobiliere-et-privatisation.html Lemieux C., 2009. Le Devoir et la grâce. Paris, Economica, coll. Etudes sociologiques, 246 p. 
Leprêtre N., 2015. Quelle action publique pour la mise en œuvre de réseaux électriques « intelligents »? Une analyse comparée des expérimentations de Smart Communities au Japon. Communication au Congrès de l'Association Française de Science Politique, Aix-en-Provence, 22 juin.

Leprêtre N., 2016. Les évolutions des politiques énergétiques japonaises face aux enjeux environnementaux et aux changements technologiques. Japan Analysis (article à paraitre).

Lorrain D., 2004. « Portrait d'entreprise » Hitachi : un industriel urbain « total ». Flux, $\mathrm{n}^{\circ} 55$, p. 60-72.

Meijer A. J., Rodriquez Bolivar M. P., 2014 (to be published). Governing the Smart City. A Review of the Literature on Smart Urban Governance. International Review of Administrative Science.

Mah D., Wu Y-Y., Ip J.C., Hills P.R., 2013. The role of the state in sustainable energy transitions: A case study of large smart grid demonstration projects in Japan. Energy Policy, 63, décembre, p. 726-737.

Muller P., 2000. L'analyse cognitive des politiques publiques : vers une sociologie politique de l'action publique. Revue Française de Science Politique 50, nº 2, p. 189-208.

Nappi-Choulet I., 2009. Les mutations de l'immobilier : de la finance au développement durable. Paris, Autrement, $304 \mathrm{p}$.

Peyroux E., 2014. International Policy Circulation and City-to-City Co-operation: Geopolitics and policy learning in Africa and the global South. Communication à la 3e Rencontre des Études Africaines en France, IEP, Université de Bordeaux, 02/07/2014.

Picon A., 2013. Smart cities : théorie et critique d'un idéal auto-réalisateur. Paris, Éditions B2, 120 p. Sassen S., 2011. Talking back to your intelligent city. Février, McKinsey \& Company - McKinsey on Society. URL : http://voices.mckinseyonsociety.com/talking-back-to-your-intelligent-city/ consulté le 7 janvier 2015.

Sennett R., 2012. The stupefying smart city. LSE Cities, décembre. URL : http://lsecities.net/media/ objects/articles/the-stupefying-smart-city/en-gb/ - consulté le 7 janvier 2015.

Shiroyama H., Kajiki S., 2013. The analysis of the transition process. Eco-Town \& Smart Community in the City of Kitakyushu. Communication au Workshop Transition Management, University of Tokyo, 12 juillet 2013.

Söderström O., Paasche T., Klauser F., 2014. Smart cities as corporate storytelling. City, Vol. 18, Issue 3, p. 307-320.

Stockwin J. A. A., 1999. Governing Japan : Divided Politics in a Major Economy. $3^{\mathrm{e}}$ édition. Oxford: Blackwell Publishers, 320 p.

Thurbon E., 2014. L'État développeur : défense du concept. Critique internationale, 63(2), p. 59-75.

\section{NOTES}

1. À titre d'exemple, les millions de compostages électroniques à l'entrée et à la sortie du métro tokyoïte grâce aux cartes SUICA génèrent des millions de données qui, traitées de façon globale, permettent de voir l'évolution de l'usage de l'ensemble des lignes de métro par des millions d'usagers. Ces données traitées globalement constituent des méta-données, ou big datas, et résultent de la compilation de données individuelles, qui sont des données simples (en l'occurrence ici, un compostage électronique). 
2. Les expressions "smart city" et "smart community" sont employées de manière interchangeable par la plupart des acteurs japonais, à l'image de la définition proposée par le New Energy Promotion Council qui les considère explicitement comme synonymes (JSCP, 2015).

3. Dans une approche cognitive des politiques publiques (Muller 2000), il aurait été aussi intéressant de développer une analyse des référentiels d'action publique entourant l'introduction des «smart cities». Voir Nicolas Leprêtre (2015) pour des éléments d'analyse s'inscrivant dans ce courant.

4. Pour Cyril Lemieux, une grammaire est "l'ensemble des règles à suivre pour agir d'une façon suffisamment correcte aux yeux des partenaires de l'action" (2000, p. 110), ou encore "l'ensemble des règles à suivre pour être reconnu, dans une communauté, comme sachant agir et juger correctement". "Une grammaire est ce qui permet aux membres d'une communauté de juger correctement, c'est-à-dire de lier correctement à des discontinuités survenant dans le monde (corps, objets, matériaux, gestes, paroles) des descriptions et d'éprouver vis-à-vis de certaines de ces descriptions un sentiment d'évidence" (2009, p. 21-23). Pour Boltanski, une grammaire est constituée de "repères normatifs de validité plus ou moins généraux, à la fois intégrés [aux] compétences cognitives [des acteurs] et inscrits dans des dispositifs (et notamment des dispositifs d'objets), enracinés dans les situations" (2006, p. 11).

5. Si ce mouvement est général dans les pays développés, l'arrivée des acteurs de la finance dans le champ de l'urbain au Japon est un peu plus tardive. Ceci est dû à deux facteurs : l'introduction tardive de la titrisation immobilière au tournant des années 2000 (Aveline, 2008) ; le recours à des banques internes aux conglomérats auxquelles participent déjà les fudôsan (comme Mitsubishi Jisho et la Bank of Tokyo-Mitsubishi UFJ).

6. Il est ainsi intéressant de voir que le parc Disney construit dans les années 1980 à Tokyo appartient à une entreprise japonaise spécialisée dans le remembrement urbain, l'Oriental Land Company (OLC). Or, l'entreprise la plus présente dans le capital de la OLC est Mitsui Fudôsan, la plus grosse immobilière du Japon, impliquée dans l'aménagement de la « smart city » Kashiwa no Ha. Mitsui est par ailleurs elle-même partenaire historique de... Toshiba, qui se retrouve ainsi mécaniquement impliquée dans Kashiwa no Ha. L'ensemble de la coalition glisse ainsi vers l'urbain par le biais du smart.

7. Cette approche consiste en une optimisation des services de transport par la donnée (bus à la demande), la réduction des embouteillages (Intelligent Transport System) et la répartition des périodes de recharge de véhicules électriques pour ne pas fragiliser le réseau électrique (en raison d'une tendance à la concentration de recharges rapides en fin de journée). Ces acteurs liés au transport sont présents depuis longtemps dans la gestion de l'urbain, mais leur association nouvelle avec les entreprises «IT » recontextualise leur poids.

8. La réforme fiscale et économique de juin 2007 les définit comme des « initiatives globales pour le développement technologique et les changements sociaux, utilisant sa dominance en hautes technologies dans le secteur des monozukuri (biens manufacturés), et dans le secteur de l'environnement et des économies d'énergie comme force motrice, dans le but de réaliser une société durable». Autrement dit, les éco-innovations sont au Japon ce que les technologies durables sont en Europe.

9. S'ajoutent trois autres programmes: les « Next Generation Energy Technology Demonstration Projects » ont été mis en place dans huit villes en 2011-2012; le programme «Projects Supporting the Wider Acceptance of Smart Community Concepts » consiste en des études de faisabilité ; enfin des «smart communities » ont été mises en œuvre dans huit villes et villages de la région dévastée par le tsunami de 2011.

10. Capitale de la préfecture de Kanagawa et voisine de Tokyo, Yokohama est la deuxième ville du Japon avec 3,7 millions d'habitants. Son projet présente la spécificité de ne pas être circonscrit à un quartier en particulier puisque la majorité des arrondissements sont concernés par 
l'expérimentation. Sa plus grande expérimentation comprend 4000 HEMS installés dans des bâtiments déjà bâtis.

11. La ville de Toyota ( 420000 habitants) est le berceau de l'entreprise automobile Toyota Motors. Située aux abords de Nagoya, sa large superficie (plus de $900 \mathrm{~km}^{2}$ ) et sa faible densité rendent ses habitants très dépendants de la voiture individuelle. Par conséquent, l'expérimentation, concentrée sur deux quartiers résidentiels, met l'accent sur la «smart mobility " et les connexions entre les véhicules de nouvelle génération, les «smart houses » et le réseau électrique (système de $\mathrm{V} 2 \mathrm{H}$, vehicle to home).

12. Keihanna se distingue par son statut de cité scientifique à la jonction des départements de Kyoto, Osaka et Nara (410 000 habitants au total), et par la focalisation de son expérimentation sur des logements individuels en lotissement résidentiel périurbain. Le projet inclut un système de DR avec des conseils personnalisés de grande ampleur (près de 700 foyers).

13. La ville de Kitakyushu est située sur l'île de Kyushu et compte environ un million d'habitants. Ancien bastion de l'industrie lourde nippone et longtemps célèbre pour son extrême niveau de pollution, Kitakyushu constitue désormais une référence en matière de reconversion écologique. Située dans le quartier d'Higashida, l'expérimentation inclut les associations environnementales locales, et repose notamment sur une production électrique hydrogène.

14. Ces requêtes prennent la forme pour l'essentiel d'une tarification dynamique, avec un changement réel du prix à Kitakyushu (de $15 \mathrm{y} / \mathrm{kWh}$ à $150 \mathrm{y} / \mathrm{kWh}$ selon l'heure d'utilisation et la force du pic) et sous la forme d'un système de points dans les trois autres Smart Communities (avec une perte ou un gain de points, restitués sous la forme d'argent à la fin de la saison).

15. Supervisory Control and Data Acquisition. Le système SCADA permet de rassembler toutes les batteries de stockage connectées en une batterie virtuelle unique avec laquelle interagira le CEMS. L'objectif est une optimisation de l'utilisation de l'ensemble des batteries de la communauté.

16. Panasonic est le concurrent direct de Toshiba pour l'établissement de normes techniques et pour l'accès au marché du «smart». Historiquement, la région du grand Tokyo (le Kantô) est un bastion industriel et économique de Toshiba, Panasonic opérant plutôt dans le Kansai (conurbation Osaka-Kyoto-Kobe). Or, Panasonic a récemment saisi l'opportunité des «smart cities " pour pénétrer le bastion de Toshiba, en y développant une «smart city " (Fujisawa Smart Town) et en pénétrant le marché du «smart » à Yokohama. Parmi les 4000 HEMS mis en place, Panasonic a réussi à fournir un plus grand nombre de HEMS aux participants volontaires que Toshiba, son partenaire et concurrent dans ce projet, au grand dam de Toshiba dont les responsables ont confié lors d'entretiens semi-directifs leur embarras.

17. Le projet de Lyon Confluence associe un smart grid pour les bâtiments de l'îlot Hikari, un bâtiment à énergie positive, un système de voitures électriques en auto-partage, une production d'énergie renouvelable, un système de visualisation de l'énergie consommée dans chaque logement et bureaux, le tout relié à un CEMS. L'objectif est de réduire la consommation d'énergie de $25 \%$ d'ici à 2020 .

\section{ABSTRACTS}

The "smart" cities constitute a new urban imaginary which has recently been spreading through the world, in particular in East Asia, North America, and Europe. Is it for all that a new urban model and, in that case, is it a unique and globalized one? The paper analyzes the "smart" 
communities, Japanese declination of "smart" cities. Rather than a model, the "smart" communities are part of what pragmatic sociology calls a grammar of the urban. If there is somehow a model, the Japanese case teaches us it might be more in the governance of this new object whom experimentations are various and heterogeneous, in particular in the context of the Japanese developmentalist regime, part of the Japanese capitalism. The exportability of the "smart" communities is therefore not the result of the globalization of models, but results from the complex strategy of private companies, such as Toshiba.

Les «smart " cities constituent un nouvel imaginaire qui se répand à travers le monde, en particulier en Asie orientale, en Amérique du Nord et en Europe. Est-on pour autant face à un nouveau modèle urbain et, si tel était le cas, à un modèle unique globalisé ? La présente contribution prend pour cas d'étude les «smart» communities, déclinaisons japonaise des «smart» cities. Plutôt que d'un modèle, les «smart» communities relèveraient de ce que la sociologie pragmatique appelle une grammaire de l'urbain. Si modèle il y a, le cas japonais nous enseigne qu'il se situerait plutôt dans la gouvernance de ce nouvel objet aux expérimentations assez variées et hétérogènes, en particulier dans le cadre du régime développementaliste, caractéristique du capitalisme japonais. L'exportabilité des «smart » communities ne relève dans ce cas pas d'une globalisation des modèles, mais plutôt d'une stratégie d'entreprise aux enjeux complexes, que l'article analyse avec le cas de Toshiba.

\section{INDEX}

Mots-clés: « Smart » cities, « smart » communities, Japon, Toshiba, modèle, régime développementaliste

Keywords: « Smart » cities, « smart » communities, Japan, Toshiba, model, developmentalist regime

Subjects: Sur le Champ - Sur le Terrain

\section{AUTHORS}

\section{RAPHAËL LANGUILLON-AUSSEL}

Raphael Languillon-Aussel, rlanguillon@gmail.com est Chercheur en post-doctorat à l'Université de Chuo - Tokyo (Japon), Boursier de la Japanese Society for the Promotion of Sciences, Boursier de la Fondation Palladio pour l'immobilier.

\section{NICOLAS LEPRÊTRE}

Nicolas Leprêtre, nicolas.lepretre@ens-lyon.fr, est Chercheur en doctorat, Attaché Temporaire d'Enseignement et de Recherche, UMR 5062 Institut d'Asie Orientale, École normale supérieure de Lyon

\section{BENOIT GRANIER}

Benoit Granier, benoit.granier@ens-lyon.fr, est Chercheur en doctorat, Attaché Temporaire d'Enseignement et de Recherche, UMR 5062 Institut d'Asie Orientale, Université de Lyon. 